\title{
DESCRIPTION OF CLASSICAL AND QUANTUM INTERFERENCE IN VIEW OF THE CONCEPT OF FLOW LINE
}

\author{
Milena Davidović, ${ }^{1}$ Ángel S. Sanz, ${ }^{2}$ and Mirjana Božićc $\hat{c}^{3 *}$ \\ ${ }^{1}$ Faculty of Civil Engineering, University of Belgrade, 11000 Belgrade, Serbia \\ ${ }^{2}$ Instituto de Física Fundamental (IFF-CSIC), Serrano 123, 28006 Madrid, Spain \\ ${ }^{3}$ Institute of Physics, University of Belgrade, 11080 Belgrade, Serbia \\ *Corresponding author e-mail: bozic@ipb.ac.rs \\ E-mails: milena@grf.bg.ac.rs, asanz@iff.csic.es
}

\begin{abstract}
Bohmian mechanics, a hydrodynamic formulation of quantum mechanics, relies on the concept of trajectory, which evolves in time in compliance with dynamical information conveyed by the wave function. Here this appealing idea is considered to analyze both classical and quantum interference, thus providing an alternative and more intuitive framework to understand the time-evolution of waves, either in terms of the flow of energy (for mechanical waves, sound waves, electromagnetic waves, for instance) or, analogously, the flow of probability (quantum waves), respectively. Furthermore, this procedure also supplies a more robust explanation of interference phenomena, which currently is only based on the superposition principle. That is, while this principle only describes how different waves combine and what effects these combinations may lead to, flow lines provide a more precise explanation on how the energy or probability propagate in space before, during and after the combination of such waves, without dealing with them separately (i.e., the combination or superposition is taken as a whole). In this sense, concepts such as constructive and destructive interference, typically associated with the superposition principle, physically correspond to more or less dense swarms of (energy or probability) flow lines, respectively. A direct consequence of this description is that, when considering the distribution of electromagnetic energy flow lines behind two slits, each one covered by a differently oriented polarizer, it is naturally found that external observers' information on the slit crossed by single photons (understood as energy parcels) is totally irrelevant for the existence of interference fringes, in striking contrast with what is commonly stated and taught.
\end{abstract}

Keywords: Bohmian mechanics, flow line, interference, sound wave, electromagnetic wave, Umov vector, Poynting vector, probability current

\section{Introduction}

Superposition and interference are two intertwined capital concepts in both classical and quantum wave mechanics. As is well known, within the framework of a general wave theory, superposition simply consists in adding two or more waves. In elementary courses on wave mechanics [1-3], it is commonly taught that when two traveling waves propagate through a certain medium, the net displacement of the 
medium at a particular point and a given time is just the direct sum of the individual displacements associated with each contributing wave.

Such a traditional conception of interference, where only the net effect of the waves is considered and the associated flow of energy is often neglected (or just pushed into the background), strongly relies on the mathematical grounds of wave mechanics, namely the superposition principle. According to this principle, given a set of field-like variables $\left\{\vartheta_{i}\right\}$, solutions to a linear flow equation

$$
\Phi \vartheta_{i}=0,
$$

where $\Phi$ denotes the (space and time) flow operator

$$
\Phi=\frac{\partial^{2}}{\partial t^{2}}-c^{2} \frac{\partial^{2}}{\partial x^{2}}
$$

and $c$ is a constant typically identified with the propagation (or diffusion) speed of the field variable, new solutions can be readily built up from linear combinations of the $\vartheta_{i}$,

$$
\Theta=\sum_{i=1}^{N} c_{i} \vartheta_{i} .
$$

This intuitive principle has an important practical advantage: it allows us to find and devise smart methods to solve linear flow equations, like Eq. (1), by just decomposing the total solution into a set of partial contributions. Besides, in our opinion it also brings in an important inconvenience: our conception of physical superposition is directly grounded on its mathematical basis, which becomes critical when Eq. (1) represents a wave equation and the field variables refer to waves propagating from different sources. As a consequence, for example, following the above mathematical roots, it is commonly thought that when two waves superimpose, they pass each other just as if each wave was unaware of the other; only the net effect matters [2]. This is just a physical model to understand and explain the wave phenomena observed in Nature. However, it also strongly determines our perception of such phenomena, what has very important consequences particularly in the case of quantum systems.

To some extent, such a picture of interference is rather poor. It refers to the superposition or combination of partial waves, although in real life these waves never appear separately. In order to get some more light on interference, let us get back to the usual notion of wave from classical wave theory, where it typically represents a perturbation that propagates throughout a certain medium. From a physical viewpoint, the propagation of a perturbation in a material medium is associated with a flow of energy from one place to another within such a medium. A better understanding of interference should therefore include the tools to determine how this flow takes place, and not only rely on how different wave components (which we never observe in real life) combine. The same idea, of course, can be naturally extended to quantum systems. Even if the nature of quantum waves is different from that of classical waves, the key element, namely the transport or flow of a certain quantity (probability in the case of the former and energy for the latter) is the same.

In this work we tackle the issue of interference within a general theoretical framework based on the concept of flow line, applicable to both classical and quantum systems. Within this scenario, while the superposition principle only tells us how different waves combine, the trajectories or flow lines provide us with information on how the net effect propagates before, during and after their combination, putting the emphasis on the more natural conception of considering the wave as a whole, and not on splitting it up in its different components, as it is typically done. 
It is worth noting that, although the idea of transversal flow is mentioned in different sources (for example, see energy flow for non-dispersive waves in [1], or for the probability flow in quantum processes in $[4,5]$ ), the idea of monitoring it during the full propagation of the wave (i.e., time by time) is not that general at all. As far as we know, the use of flow lines as a working tool to visualize the propagation of energy in sound waves can be traced back to the 1980s 6 10]. The concept of flow line is quite general and can be found in many different physical contexts as well [11], including classical electromagnetic problems (see Ref. 12 and references therein). Specifically, in most of these works this concept is introduced after assuming an analogy between the corresponding problem and a hydrodynamic one.

Together with the fact that flow lines constitute an interesting working tool to analyze the evolution (flow) of energy or probabilities, we would also like to stress that depending on the problem considered, these elements present the nice feature that they can be somehow inferred from experimental data, as shown recently in the case of Young's two-slit experiment [13]. This representation is in compliance with the recent method devised to determine the photon wave function [14], based on the so-called weak-measurement technique [15], which constitutes a remarkable alternative to the more traditional construction of phase-space tomograms [16].

This paper is organized as follows. In Sec. 2 we discuss sound waves as an example of classical waves, introducing the associated equation for the energy flow lines. As an illustration, we present a simulation of the energy flow lines behind a wall with two openings. In this example, the nodes in the eventual fringe pattern represent regions of silence (or, to be more precise, low sound) in between regions where the sound is reinforced. This experience could be equally reproduced with two synchronized loudspeakers emitting the same sound pitch. The homologous equation for electromagnetic energy (EME) flow lines is given in Sec. 3. Moreover, in analogy with the previous case, in this section we also show the EME flow lines behind two slits, although each slit is followed by a linear polarizer with its polarization axis oriented in a different direction. In Sec. 4 we discuss how an even more general picture of interference can be achieved for quantum nonzero-mass particles after considering flow lines of quantum-mechanical probability density. Finally, the main conclusions arising from this work are summarized in Sec. 5 .

\section{Sound Energy Flow Lines: Interference behind Two Openings}

In order to derive the equation of motion accounting for the transport of energy of a sound wave propagating through a non-viscous fluid, we start from the continuity and momentum equations [3, 17, 18],

$$
\begin{aligned}
\frac{\partial \rho}{\partial t}+\nabla(\rho \mathbf{v}) & =0 \\
\rho \frac{\partial \mathbf{v}}{\partial t}+\nabla p+\rho \mathbf{v} \cdot \nabla \mathbf{v} & =0
\end{aligned}
$$

respectively, where $\rho(\mathbf{r}, t)$ is the fluid density, $\mathbf{v}(\mathbf{r}, t)$ is the velocity of a fluid element, and $p(\mathbf{r}, t)$ is the pressure. Assuming the velocity of the fluid and the fluctuations of the density and pressure are small $\left(\rho=\rho_{0}+\rho^{\prime}, \rho^{\prime} \ll \rho_{0}, p=p_{0}+p^{\prime}, p^{\prime} \ll p_{0}, \mathbf{v}=\mathbf{v}^{\prime}\right)$, after linearization the above equations become

$$
\begin{gathered}
\frac{\partial \rho^{\prime}}{\partial t}+\rho \nabla \mathbf{v}^{\prime}=0 \\
\rho_{0} \frac{\partial \mathbf{v}^{\prime}}{\partial t}+\nabla p^{\prime}=0 .
\end{gathered}
$$


Taking into account the relationship between pressure and density,

$$
\left.\frac{\partial p}{\partial \rho}\right|_{\rho=\rho_{0}}=\frac{p^{\prime}}{\rho^{\prime}}=c^{2} .
$$

where $c$ is the speed of sound in the corresponding medium, from Eqs. (6) and (7) we readily obtain the wave equation for the acoustic pressure,

$$
\frac{1}{c^{2}} \frac{\partial^{2} p^{\prime}}{\partial t^{2}}-\nabla^{2} p^{\prime}=0
$$

The energy transport in the sound field is described by the equation

$$
\frac{\partial}{\partial t}\left(\frac{1}{2} \rho_{0} v^{\prime 2}+\frac{p^{2}}{2 \rho_{0} c^{2}}\right)+\nabla\left(p^{\prime} \mathbf{v}^{\prime}\right)=0
$$

where

$$
\mathbf{S}=p^{\prime} \mathbf{v}^{\prime}
$$

is the so-called Umov energy flow vector $[3,19]$, the mechanical equivalent of the Poynting or PoyntingHeaviside vector, commonly used in electromagnetism [20].

Let us now consider without loss of generality and for simplicity a sound wave of constant angular frequency $\omega$ (the analysis could be carried out as well with non-monochromatic waves). If the pressure and velocity are taken as complex quantities (a typical working technique when dealing with wave equations, although only the real part of these quantities is physically meaningful),

$$
p^{\prime}=P e^{-i \omega t}, \quad \mathbf{v}^{\prime}=\mathbf{V} e^{-i \omega t}
$$

after substitution into Eqs. (7) and (8), we have

$$
\begin{array}{r}
\nabla^{2} P+\left(\frac{\omega}{c}\right)^{2} P=0, \\
i \omega \mathbf{V}=\frac{\nabla P}{\rho_{0}} .
\end{array}
$$

In the case of high-frequency waves, to avoid fast oscillations, we can consider time-averaging over a cycle, which allows us to introduce the definition of the time-averaged energy flow vector,

$$
\langle\mathbf{S}\rangle=\frac{1}{2} \operatorname{Re}\left\{P \mathbf{V}^{*}\right\}
$$

The modulus of this vector determines the sound intensity [17],

$$
I=|\langle\mathbf{S}\rangle|,
$$

related to the energy density $w$ as

$$
w=\frac{I}{c}=\frac{|\langle\mathbf{S}\rangle|}{c} .
$$

Consider now the propagation of the above monochromatic plane sound wave incident onto an obstacle with two slits, located on the $X Z$ plane. Assuming the amplitude of the incident complex pressure does 


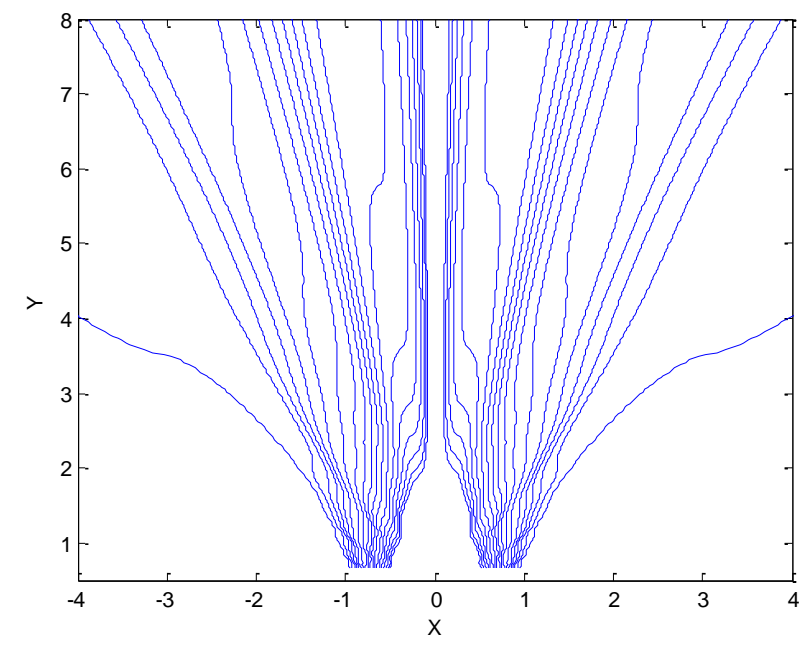

Figure 1: Energy flow lines associated with sound waves emerging from an obstacle with two slits. The width of the openings is $\delta=0.5 \mathrm{~m}$, their mutual distance $d=1.5 \mathrm{~m}$, the speed of sound $c=340 \mathrm{~m} / \mathrm{s}$, and its frequency $f=1 \mathrm{kHz}$.

not depend on the $\mathrm{z}$ coordinate and has the functional form $P(x) e^{i k y}$, the solution of the Helmholtz equation (13) behind the obstacle can be expressed in terms of the well-known Fresnel-Kirchhoff integral from the theory of propagation of optical waves 20,22 ,

$$
P(x, y)=e^{-i \pi / 4} e^{i k y} \sqrt{\frac{k}{2 \pi y}} \int_{-\infty}^{\infty} P\left(x^{\prime}, 0\right) e^{i k\left(x-x^{\prime}\right)^{2} / 2 y} d x^{\prime} .
$$

Here, $P\left(x^{\prime}, 0\right)$ is the initial pressure, which in our case is given by the pressure just behind the obstacle, determined by the corresponding boundary conditions. From Eqs. (14) and (15), we thus obtain

$$
\langle\mathbf{S}\rangle=-\frac{1}{2 \rho_{0} \omega} \operatorname{Im}\left\{P \frac{\partial P^{*}}{\partial x} \mathbf{e}_{x}+P \frac{\partial P^{*}}{\partial y} \mathbf{e}_{y}\right\}
$$

where $\mathbf{e}_{x}$ and $\mathbf{e}_{y}$ are unit vectors along the $x$ and $y$ directions. The vector field $\langle\mathbf{S}\rangle$ is tangent to the sound energy flow lines at each point determined by the equation

$$
\frac{d \mathbf{r}}{d r}=\frac{\langle\mathbf{S}\rangle}{I} .
$$

Behind a one-dimensional grating, this equation can be recast as

$$
\frac{d y}{d x}=\frac{\left\langle S_{y}\right\rangle}{\left\langle S_{x}\right\rangle}
$$

Sound energy flow lines calculated from Eqs. (18)-(21) for a totally absorptive obstacle with two identical openings are shown in Fig. 1. We find that the energy flow lines associated with different slits do not cross the system symmetry line. This means that there is a kind of fictitious barrier between the 
two sound sources (slits). The flux-line non-crossing property for classical/sound waves is analogous to the Bohmian non-crossing property 23 25]. This correspondence can be set on the basis of the analogy between Eq. 20 and the equation of trajectories determined by the quantum probability current density (see Sec. 4 for a more detailed discussion).

It is worth noticing that sound waves are described by field variables, i.e., continuous functions of space and time, although the gases, liquids or solids through which these waves propagate consists of individual, discrete objects (e.g., atoms, ions, molecules, etc.). However, in the case of fluids, for instance, instead of appealing to these objects to analyze the corresponding hydrodynamics, it is common to make use of tracer particles, which help to elucidate the flow dynamics and hence the dynamical properties of the fluid 26,27$]$.

The propagation of sound waves (energy) is of practical interest, particularly in room acoustics [28]. In this area, sound energy flow lines have been evaluated in the past using different approximate methods. Recently, the diffraction of these waves at half planes and slits has been studied 29, 30 by means of methods of scalar optics in the far field. The method described above also makes use of results from optics (in general, electromagnetism), although is more general, since it encompasses both near and far field.

\section{Electromagnetic Energy Flow Lines: Interference behind Two Slits Covered by Polarizers}

Can the electromagnetic field associated with light be thought as a kind of "wave function for the photon"? Although this is a longstanding challenging question, it is still of much interest nowadays. Scully and Zubairy summarized [31 the objections posed by Kramers [32, Power [33 and Bohm [34 against the concept of a photon, agreeing with Kramers and Bohm that the concept of a photon wave function should be carefully used, since it can be very misleading. Nonetheless, they concluded that each objection can be overcome, suggesting a path to circumvent them, and providing a way to construct the photon wave function through the radiation-field second quantization formulation.

Raymer and Smith [35, Bialinicki-Birula 36 38] and Holland 39] went a step further. Holland [39] analyzed similarity of Maxwells' equation with quantum wave equations (Schrodinger's and Dirac's). Raymer and Smith [35] and Bialinicki-Birula [36 38] consider that the usual electromagnetic Maxwell field is the quantum wave function of the single photon. The fact that it transforms like a threedimensional vector arises from the spin-one nature of the photon. The interpretation of the Maxwell field is, therefore, akin to the Schrdinger wave function, which describes the evolution of probability amplitudes associated with various possible quantum events in which a particle's position is found to be within a certain volume. In this sense, Maxwell's equations rule the evolution of probability amplitudes for various possible quantum events in which the photon's energy is found within a certain volume.

Assuming this interpretation for the Maxwell field, and based on a previous work by Prosser [40, 41], a method to determine the electromagnetic energy flow lines behind various gratings illuminated by a monochromaticbeam of light was developed in [12,42]. In this case, the solutions of Maxwell's equations behind the gratings were determined using the solution of the Helmholtz equation. (It is well known that the space-dependent part of each component of the electric $\mathbf{E}(\mathbf{r})$ and magnetic $\mathbf{H}(\mathbf{r})$ free fields satisfy Helmholtz's equation).

As before, again here we are going to consider the time-averaged energy flux vector to determine the electromagnetic energy flow lines (for a non-averaged, time-dependent application, see the work by Chou 
and Wyatt [4]), given by the real part of the complex Poynting vector 44],

$$
\mathbf{S}(\mathbf{r})=\operatorname{Re}\left[\frac{1}{2} \mathbf{E}(\mathbf{r}) \times \mathbf{H}^{*}(\mathbf{r})\right]
$$

Since the energy flow is along the direction of the Poynting vector, the EME flow lines will be determined from the parametric differential equation

$$
\frac{d \mathbf{r}}{d s}=\frac{\mathbf{S}(\mathbf{r})}{c U(\mathbf{r})}
$$

where $s$ is a certain arc-length and $U(\mathbf{r})$ is the time-averaged electromagnetic energy density

$$
U(\mathbf{r})=\frac{1}{4}\left[\epsilon_{0} \mathbf{E}(\mathbf{r}) \cdot \mathbf{E}^{*}(\mathbf{r})+\mu_{0} \mathbf{H}(\mathbf{r}) \cdot \mathbf{H}^{*}(\mathbf{r})\right] .
$$

As mentioned in the previous section, the Poynting vector in electromagnetism plays the same role as the Umov vector (19) in the theory of mechanical waves. Since in the last instance both vectors are just transport vectors, some authors use the combined denomination Poynting-Umov vector [3] (or also Heaviside-Poynting-Umov vector, to include Heaviside's contribution).

Holland argued [39] that, for reasons of compatibility with quantum mechanics, it does not seem to be reasonable assuming that the tracks $\mathbf{r}=\mathbf{r}(t)$ deduced from the equation essentially equivalent to Eq. (23) can be identified with the orbits of "photons". However, Ghose et al. showed [45] that Bohmian trajectories for relativistic bosons, and so for photons, could be defined indeed. They derived an equation of motion for massless bosons, which is equivalent to Eq. (23). From such an equation they obtained photon trajectories behind the two-slit grating, assuming that the wave function at the point $(y, x)$, at the sufficient distance $D \gg d^{2} / \lambda$ to the right of the plane of the slits, is a superposition of two spherical waves.

EME flow lines evaluated from Eq. (23) in [12 42 show that the energy redistribution behind a multiple slit grating corresponds with a Talbot pattern in the near field, and with a Fraunhofer interference pattern in the far field. The Fresnel-Arago laws as well as the Poisson-Arago spot were also interpreted using EME flow lines [46, 47]. The main conclusion from the analysis of these phenomena is that the motion of an eventual photon wave packet essentially represents the flow of electromagnetic energy along an ensemble of flow lines.

Actually, EME flow lines obtained [47,48] from a numerical simulation of Young's two-slit experiment with parameters taken from the experiment performed by Kocsis et al. [13] showed a good agreement with the averaged photon trajectories inferred from the experimental data. It is worth stressing that the photon trajectories reconstructed from the experiment were in compliance with the Bohmian approach, thus confirming that trajectories coming from different slits do not cross. This means that, at the level of the average electromagnetic field (or the wave function, in the case of material particles, in general), full which-way information can still be inferred without destroying the interference pattern. That is, rather than complementarity, the experiment seems to suggest that a photon wave function has a tangible (measurable) physical reality [48], in agreement with a recent theorem on the realistic nature of the wave function 49 .

Let us now evaluate the EME flow lines behind two slits in a more general case, when each slit is covered by a polarizer with its polarization axis oriented at a different angle. In our model, we consider a monochromatic electromagnetic wave in vacuum, traveling along the $y$ direction, incident onto a two-slit grating located on the $X Z$ plane, at $y=0$, with infinitely long slits parallel to the $z$ axis, so that both 
the electric and magnetic fields do not depend on the $z$ coordinate. The electric and magnetic fields of the incident wave are given by the expressions

$$
\begin{aligned}
\mathbf{E}_{\mathrm{inc}} & =A e^{i k y} \mathbf{e}_{z}-B e^{i \varphi} e^{i k y} \mathbf{e}_{x} \\
\mathbf{H}_{\mathrm{inc}} & =\sqrt{\frac{\epsilon_{0}}{\mu_{0}}} B e^{i \varphi} e^{i k y} \mathbf{e}_{z}+\sqrt{\frac{\epsilon_{0}}{\mu_{0}}} A e^{i k y} \mathbf{e}_{x} .
\end{aligned}
$$

Since the slits are followed by polarizers, with their polarization axis at angles $\theta_{1}$ and $\theta_{2}$ with respect to the $z$ axis, the $z$ components of the fields behind the grating read as

$$
\begin{aligned}
\mathbf{E}_{z}(\mathbf{r})= & A \cos ^{2} \theta_{1} \psi_{1}(\mathbf{r})+A \cos ^{2} \theta_{2} \psi_{2}(\mathbf{r}) \\
& -B e^{i \varphi} \sin \theta_{1} \cos \theta_{1} \psi_{1}(\mathbf{r})-B e^{i \varphi} \sin \theta_{2} \cos \theta_{2} \psi_{2}(\mathbf{r}), \\
\mathbf{H}_{z}(\mathbf{r})= & -\sqrt{\frac{\epsilon_{0}}{\mu_{0}}} A \cos \theta_{1} \sin \theta_{1} \psi_{1}(\mathbf{r})-\sqrt{\frac{\epsilon_{0}}{\mu_{0}}} A \cos \theta_{2} \sin \theta_{2} \psi_{2}(\mathbf{r}) \\
& +\sqrt{\frac{\epsilon_{0}}{\mu_{0}}} B e^{i \varphi} \sin ^{2} \theta_{1} \psi_{1}(\mathbf{r})+\sqrt{\frac{\epsilon_{0}}{\mu_{0}}} B e^{i \varphi} \sin ^{2} \theta_{2} \psi_{2}(\mathbf{r}) .
\end{aligned}
$$

Where $\psi_{1}(\mathbf{r})$ and $\psi_{2}(\mathbf{r})$ are scalar functions that satisfy the Helmholtz equation and the boundary conditions at the slits. The functions $\psi_{1}(\mathbf{r})$ and $\psi_{2}(\mathbf{r})$ can be recast in the form of a Fresnel-Kirchhoff integral,

$$
\psi_{i}(x, y)=\frac{k}{2 \pi y} e^{-i \pi / 4} e^{i k y} \int_{-\infty}^{\infty} \psi_{i}\left(x^{\prime}, 0^{+}\right) e^{i k\left(x-x^{\prime}\right)^{2} / 2 y} d x^{\prime}
$$

with $i=1,2$, and where the $\psi_{i}\left(x^{\prime}, 0^{+}\right)$denote their value just behind the first and the second slits, respectively, and in front of the polarizers.

Since the electric and magnetic fields do not depend on the $z$ coordinate, from Maxwell's equations one obtains two independent sets of equations [20]: one involving the $H_{x}$ and $H_{y}$ components of the magnetic field and the $E_{z}$ component of the electric field (commonly referred to as $E$-polarization),

$$
\frac{\partial E_{z}}{\partial y}=\frac{i \omega}{\epsilon_{0} c^{2}} H_{x}, \quad \frac{\partial E_{z}}{\partial x}=-\frac{i \omega}{\epsilon_{0} c^{2}} H_{y}
$$

and another involving $E_{x}, E_{y}$ and $E_{z}$ ( $H$-polarization),

$$
\frac{\partial H_{z}}{\partial y}=-i \omega \epsilon_{0} E_{x}, \quad \frac{\partial H_{z}}{\partial x}=i \omega \epsilon_{0} E_{y}
$$

From Eqs. (27) and (28) we can readily calculate the E-polarized component of the field. Similarly, from Eqs. (30) and (31) we will obtain the H-polarized component of the field behind the grating. Once we have these expressions, their substitution into Eqs. (22) and (23) leads us to the corresponding EME flow lines.

In Figs. 2 and 3 we can observe a series of sets of EME flow lines for different values of the polarization angles $\theta_{1}$ and $\theta_{2}$ (the numerical details of the simulations are provided in the corresponding captions), where the wave function just behind each slit, and before they are acted by the polarizers, is given by a Gaussian function:

$$
\psi\left(x^{\prime}, 0^{+}\right)=\psi_{1}\left(x^{\prime}, 0^{+}\right)+\psi_{2}\left(x^{\prime}, 0^{+}\right)
$$


(a)
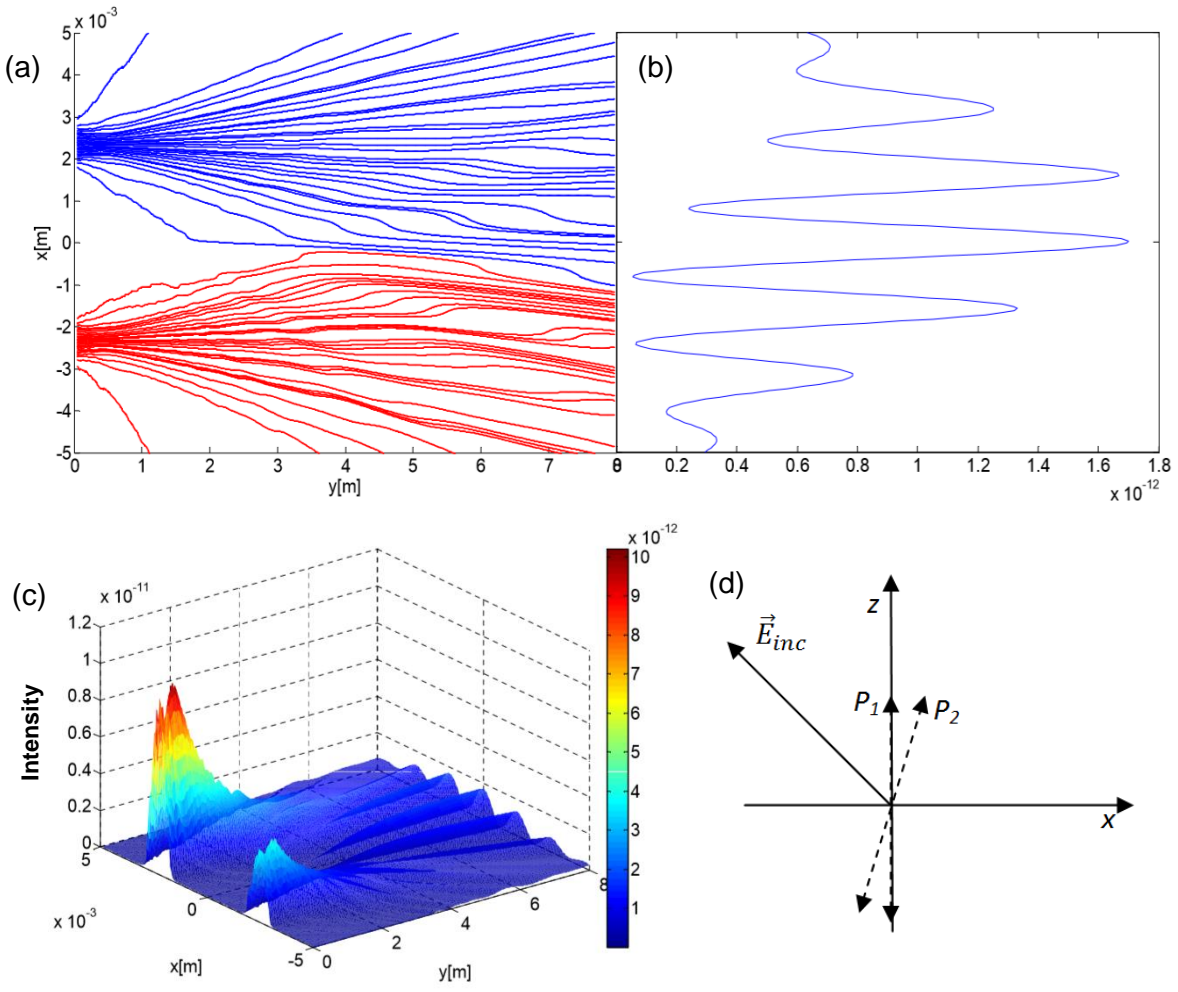

(d)

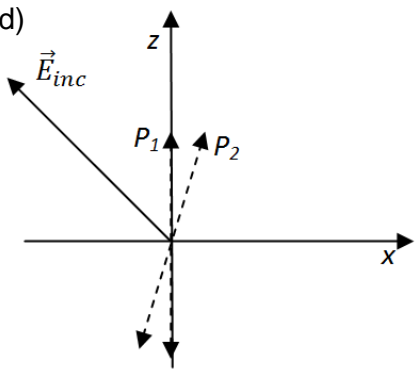

Figure 2: EME flow lines (a) and intensity distribution (b,c) behind two Gaussian slits followed by two polarizers and illuminated by monochromatic light with a wavelength $\lambda=943 \mathrm{~nm}$. The parameters considered in our simulation have been taken from the experiment data [13: $\sigma_{1}=\sigma_{2}=0.3 \mathrm{~mm}$, $\mu_{1}=-\mu_{2}=2.35 \mathrm{~mm}$, and $a_{1}=a_{2}=1.8 \sigma_{1}$, and $\lambda=943 \mathrm{~nm}$. The initial polarization is assumed to be linear, with $A=B, \varphi=0, \theta_{1}=0$, and $\theta_{2}=\pi / 10$ (d).

where

$$
\psi_{i}\left(x^{\prime}, 0^{+}\right)=\left(2 \pi \sigma_{i}^{2}\right)^{-1 / 4} e^{-\left(x^{\prime}-\mu_{i}\right)^{2} / 4 \sigma_{i}^{2}} w\left(x^{\prime}-\mu_{i}, a_{i}\right),
$$

with $i=1,2$ and $w(x, a)$ being the window function defined as

$$
w(x, a)=\left\{\begin{array}{lc}
1, & x \in[-a, a] \\
0, & \text { everywhere else }
\end{array}\right.
$$

In Fig. 2(a) we notice that the EME flow lines gives rise to a more complete description of interference phenomena compared to the description only based on intensity curves (see Fig. 2(b)). Specifically, the flow lines provide us with an idea on how the electromagnetic field propagates through space, while the intensity curves (see Fig. 2(b)) only tell us how much intensity is present at each point. In this sense, notice how in the far field the maxima and minima displayed by the light intensity correspond, respectively, to maximum and minimum values of the density of trajectories. Actually, a slight variation in the relative orientation of the axes of the polarizers behind the slits gives rise to non-vanishing minima, as seen in right panel of Fig. 2(a). Only if the axes of the polarizers are oriented along the same direction, the 
distribution of EME flow lines is symmetrical with respect to the symmetry axis (see Fig. 3(a)). However, as their relative orientation increases, as seen in the case displayed in Fig. 2 or in Figs. 3(b) and (c), the flow-line distribution is not symmetrical and total fringe visibility (vanishing minima) disappears. In this regard, the most remarkable case takes place for mutually orthogonal polarizers (see Fig. 3(b)), when the typical oscillations of intensity disappear. It is in this case when we talk about lack of interference. As it can be seen in any of the figures, the distribution of EME flow lines is in compliance with this form of intensity -it is uniform, not showing variations (see Fig. 2(b)). Apart from the fringe visibility decrease, it can also be seen a certain phase shift, downwards for conditions before orthogonality (see Fig. 2(a)) and upwards after it (see Fig. 3(c)).

In order to check the theoretically drawn trajectories presented at Figs. 2 and 3 , we propose to experimentally determine the average photon paths by adding differently oriented polarizers behind the slits to the experimental setup reported in 13 . This is a generalization of the previous proposal by Davidović et al. 47] to add orthogonal polarizers in the setup.

We consider that the proposed experiment could contribute to settle the controversy about the influence of a potential observer on the form of the interference pattern of two beams with different polarizations. The standard interpretation given to the disappearance of interference after inserting mutually orthogonal polarizers after the slits is usually based on the Copenhagen notion of the external observer's knowledge (information) about the photon paths, i.e., the slit traversed by the photon on its way to the detection screen. Proponents of the principle of complementarity affirm that information on the path destroys the interference

Using EME-flow lines, determined from the EM field and the Poynting vector, one arrives at another interpretation. One observes that EME-flow lines starting from slit 1 end up on the left-hand side of the screen, while those starting from slit 2 end up on the right-hand side, both with presence of interference and with no interference fringes. However, the distribution of EME flow lines is different in each case. For the same orientation of the polarizers, the distribution shows interference fringes (see Fig. 3(a)), while for orthogonal orientations fringes are absent (see Fig. 3(b)), in full agreement with the Arago-Fresnel laws [46. Hence, the observer's information on the slit crossed by individual photons (understood as energy parcels) seems to be totally irrelevant regarding existence of interference. What really matters is the form of the EME field, which eventually influences both the distribution and the topology of the trajectories. For values of the mutual angle between polarizers with the interval the symmetry of the interferometer setup is broken. Consequently, the intensity distribution is not symmetrical (see Figs. 2(a) and (b)). Accordingly, the distribution of trajectories is not symmetrical either, as seen in Figs. 2 (a) and 3(c). Furthermore, a certain number of trajectories starting at slit 1 (covered by a polarizer that transmits more energy than the polarizer allocated behind slit 2) cross the symmetry axis, ending up on the right-hand side (see Fig. 2(a)). Conversely, a certain number of trajectories starting at slit 2 may cross the symmetry axis, ending up on the left-hand side, if the polarizer allocated behind this slit transmits more energy than the polarizer behind slit 1, as seen in Fig. 3(c).

\section{Quantum Particle Trajectories - Flow Lines of Quantum-Mechanical Currents}

As it has been pointed out, sound waves are described in the framework of mechanics of continuum, even though the medium through which they propagate is composed of discrete objects, such as atoms or molecules, for instance. In this sense, the pressure, as a fundamental physical quantity in the description 
(a)
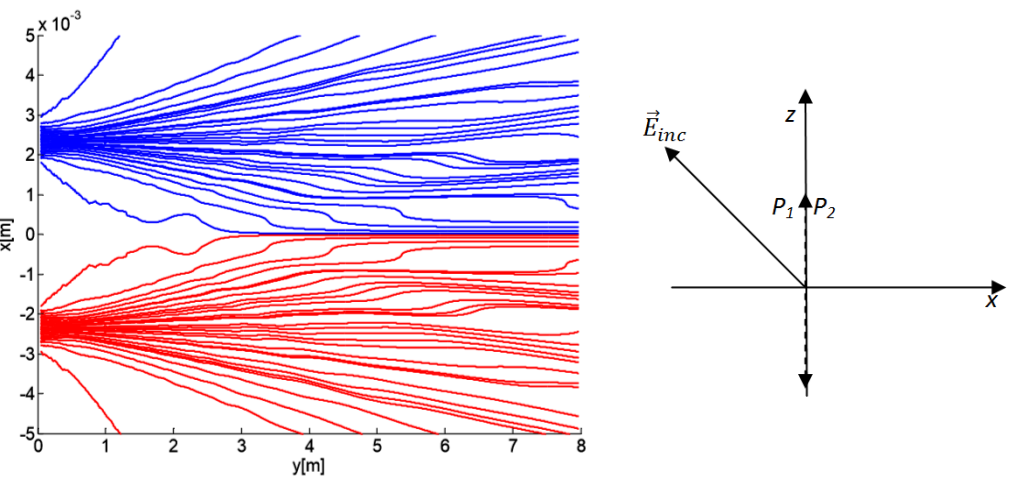

(b)
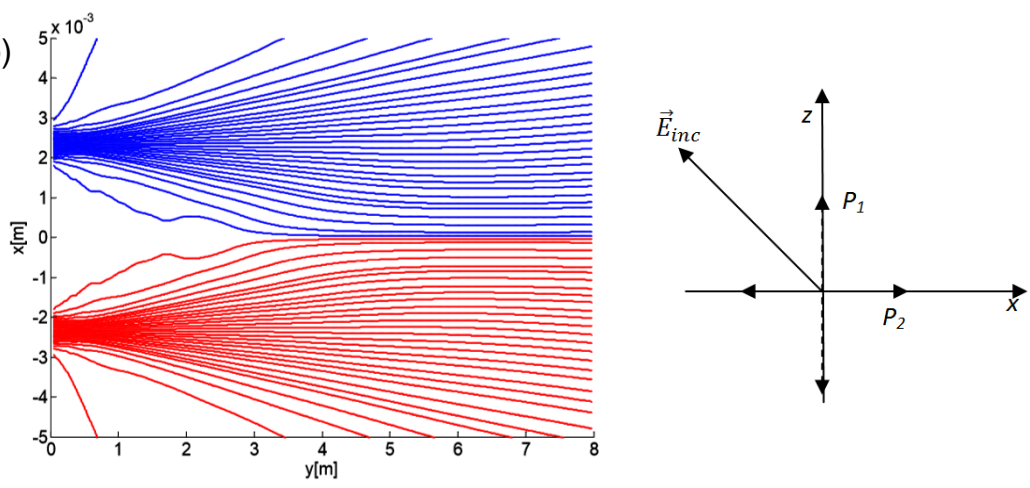

(c)
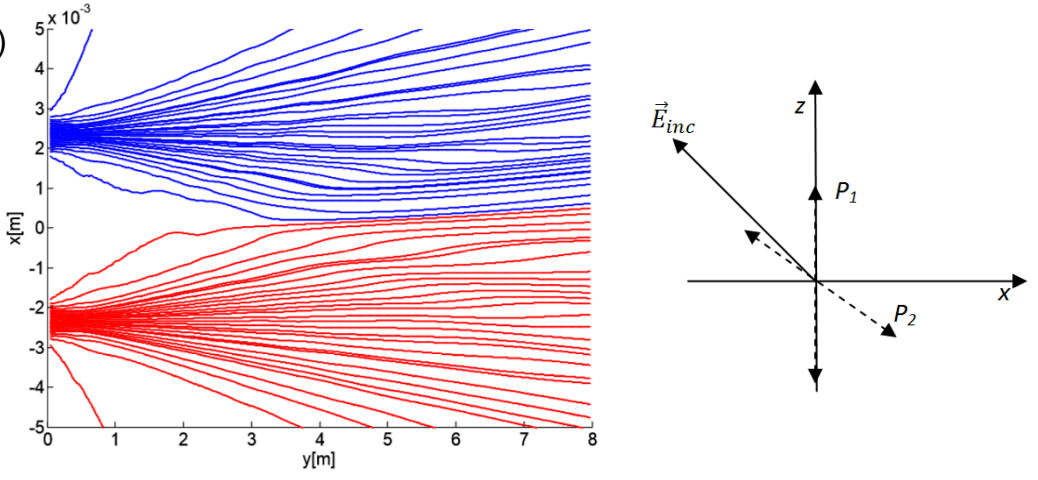

Figure 3: EME flow lines behind two Gaussian slits with the same parameters as in Fig. 2 and polarization angle $\theta_{1}=0$ and $\theta_{2}=0(\mathrm{a}), \theta_{1}=0$ and $\theta_{2}=\pi / 2(\mathrm{~b})$, and $\theta_{1}=0$ and $\theta_{2}=7 \pi / 10$ (c).

of sound waves, is a continuous function of space and time. This description is well accepted and experimentally verified. Nobody opposes or criticizes such a combination of discreteness and continuity.

In quantum mechanics, wave function $\Psi(\mathbf{r}, t)$ synthesizes continuity (wave properties) and discreteness (particle properties) in the quantum realm. But, differently from pressure in classical hydrodynamics, the physical meaning of the wave function has been open to debate since the very inception of quantum mechanics (and, by extension, also quantum optics). What is the physical meaning of a wave function associated with an individual electron, neutron, atom, molecule or photon? This is a longstanding question that has been and is still looking for an answer. 
It is within this context where we consider very useful taking into account the direct analogy between the quantum mechanical current density,

$$
\mathbf{J}(\mathbf{r}, t)=\frac{\hbar}{2 i m}\left[\Psi(\mathbf{r}, t) \nabla \Psi^{*}(\mathbf{r}, t)-\Psi^{*}(\mathbf{r}, t) \nabla \Psi(\mathbf{r}, t)\right]=|\Psi(\mathbf{r}, t)|^{2} \mathbf{v}(\mathbf{r}, t),
$$

and the Umov vector for sound waves, described by Eq. (11), on the on hand, and Poynting vector for electromagnetic waves, defined by Eq. (23), on the other hand.

A more complete or, at least, more robust picture of sound wave interference is obtained by considering flow lines determined by the Umov vector 29, 30]. The same happens in the case of photon interference when considering electromagnetic energy flow lines [12, 42, determined by the Poynting vector (23). Analogously, a more complete picture of quantum interference with massive particles arises after considering flow lines of quantum mechanical probability density $24,25,27,34,39,42,47,50,52]$. These more complete pictures might contribute to the resolutions of dilemmas and paradoxes related to the question of the physical meaning of the photon wave function as well as for the quantum mechanical wave function of nonzero mass particles.

Using quantum mechanical current density one obtains an objective interpretation of interference phenomena as a process of accumulation of single particle events, as confirmed in experiments with beams of one per one, electron [53], neutron [54] atom and molecule [55]. Intensity curves evaluated by taking modulus square of a wave function describe only the final distribution, obtained after accumulation of many particles. Intensity curves do not explain the distributions of tracks of a small number of quantum objects.

\section{Concluding Remarks}

The use of flow lines allows us to get a more complete understanding of wave propagation and interference phenomena in classical as well in quantum physics. This follows from the simulations and analyses of the flow lines associated with sound waves and electromagnetic field in a typical interference device, namely a double slit grating. In this way, larger or smaller values of flow-line densities are directly related to the common notions associated with the superposition principle of constructive and destructive interference, respectively.

In our opinion, the combined analysis of the propagation and the evolution of flow lines for sound waves will be helpful in the long standing debate about the interpretation of the quantum mechanical wave function in spite of the different nature of these two kinds of waves. Due to the analogy between classical and quantum interference phenomena, the ability to perform in a simpler fashion experiments with classical waves that mimic quantum behaviors, should render important insight on quantum systems. Notice that sound waves are described by variables that are continuous functions of space and time, although gases and fluids, which constitute the material substrate along which the wave propagates, are composed of individual, discrete objects, e.g., atoms and molecules. In the same way, trajectories in the quantum wave function do not necessarily need to account for the individual motion of a (quantum) particle, but provide us information on how probabilities flow in configuration space, and therefore how averaged swarms of identical particles travel throughout such a space.

Based on the results presented here and the recent experiment performed by Kocsis et al. 13], we would also like to propose measuring average photon trajectories behind a two-slit grating covered by two polarizers for various mutual angles between their polarization axes. We expect that this experiment 
should render trajectories in compliance with the EME flow lines displayed in Figs. 2 and 3 . Experiments of this kind should contribute to render some light on the longstanding debate on the influence of a potential observer on the form of the interference pattern of two beams with different polarizations.

\section{Acknowledgments}

Support from the Ministry of Education, Science and Technological Development of Serbia under Projects No. OI171005 (MB), OI171028 (MD) and III45016 (MB, MD), and the Ministerio de Economía y Competitividad (Spain) under Project No. FIS2011-29596-C02-01 (AS) as well as a "Ramón y Cajal" Research Fellowship with Ref. RYC-2010-05768 (AS) is acknowledged.

\section{References}

[1] I. G. Main Vibrations and Waves in Physics, Cambridge University Press, Cambridge (1993) 3rd Ed.

[2] J. D. Cutnell and K. W. Johnson Physics, John Wiley \& Sons, New York (1995).

[3] I. E. Irodov, Volnovye Procesi, Laboratoriya Bazovyh znanii, Yunimedia Stail, Moscow (2002).

[4] J. Z. H. Zhang, Theory and Application of Quantum Molecular Dynamics World Scientific, Singapore (1999).

[5] D. J. Tannor, Introduction to Quantum Mechanics. A Time-Dependent Perspective University Science Books, Sausalito, CA (2007).

[6] R. V. Waterhouse, T. W. Yates, D. Feit, and Y. N. Liu, J. Acoust. Soc. Am. 78, 758 (1985).

[7] R. V. Waterhouse and D. Feit, J. Acoust. Soc. Am. 80, 681 (1986).

[8] E. A. Skelton and R. V. Waterhouse, J. Acoust. Soc. Am. 80, 1473 (1986).

[9] R. V. Waterhouse, D. G. Crighton, and J. E. Ffowcs-Williams, J. Acoust. Soc. Am. 81, 1323 (1987).

[10] R. V. Waterhouse, J. Acoust. Soc. Am. 82, 1782 (1987).

[11] A. S. Sanz, J. Phys.: Conf. Ser. 504, 012028 (2014).

[12] A. S. Sanz, M. Davidović, M. Božić, and S. Miret-Artés, Ann. Phys. 325, 763 (2010).

[13] S. Kocsis, B. Braverman, S. Ravets, M. J. Stevens, R. P. Mirin, L. K. Shalm, and A. M. Steinberg, Science 332, 1170 (2011).

[14] J. S. Lundeen, B. Sutherland, A. Patel, C. Stewart, and C. Bamber, Nature 474, 188 (2011).

[15] Y. Aharonov, D. Z. Albert, and L. Vaidman, Phys. Rev. Lett. 60, 1351 (1988).

[16] A. Ibort, V. I. Man'ko, G. Marmo, A. Simoni, and F. Ventriglia, Phys. Scr. 79, 065013 (2009).

[17] H. Kuttruff, Acoustics: An Introduction, Taylor \& Francis, New York (2007). 
[18] A. F. Nikiforov, Lekcii po Uravneniyami Metodam Matematicheskoi Fiziki, Izdatelskii Dom Intellekt, Dolgoprudnyi (2009).

[19] N. A. Umov, Z. Math. Phys. 19, 97 (1874).

[20] M. Born and E. Wolf, Principles of Optics, Wiley, New York (1999) 7th Ed.

[21] D. Arsenović, M. Božić, O. V. Man’ko, and V. I. Man'ko, J. Russ. Laser Res. 26, 94 (2005).

[22] P. Ya. Ufimtsev, Fundamentals of the Physical Theory of Diffraction John Wiley \& Sons, Hoboken, NJ (2007).

[23] A. S. Sanz and S. Miret-Artés, J. Phys. A 41, 435303 (2008).

[24] A. S. Sanz and S. Miret-Artés, A Trajectory Description of Quantum Processes. I. Fundamentals, Lecture Notes in Physics, Springer, Berlin (2012), Vol. 850.

[25] A. S. Sanz and S. Miret-Artés, A Trajectory Description of Quantum Processes. II. Applications, Lecture Notes in Physics, Springer, Berlin (2014), Vol. 831.

[26] A. S. Sanz, An account on quantum interference from a hydrodynamical perspective, in Quantum Trajectories, K. H. Hughes and G. Parlant (Eds.), CCP6, Daresbury, UK (2011).

[27] A. S. Sanz and S. Miret-Artés, Am. J. Phys. 80, 525 (2012).

[28] H. Kuttruff, Room acoustics, Taylor \& Francis, New York (2000) 4th Ed.

[29] A. Billon and J.-J. Embrechts, Proceedings of the Acoustics 2012 Nantes Conference, pp. 2385-2390 (2012); http://hdl.handle.net/2268/119352

[30] A. Billon and J.-J. Embrechts, Acta Acustica united with Acustica 99, 260 (2013).

[31] M. O. Scully and M. S. Zubairy, Quantum Optics, Cambridge Universtiy Press, Cambridge, UK (1997).

[32] H. A. Kramers, Quantum Mechanics, North-Holland, Amsterdam (1958).

[33] E. A. Power, Introductory Quantum Electrodynamics, Longman, London (1964).

[34] D. Bohm, Quantum Theory, Prentice-Hall, Englewood Cliffs, NJ (1951) (reprinted version: Dover, New York (1989)).

[35] M. G. Raymer and B. J. Smith, Proc. SPIE 5866, 1 (2005).

[36] I. Bialinicki-Birula, Acta Phys. Pol. 34, 845 (1995).

[37] I. Bialinicki-Birula, Photon wave function, in Progress in Optics XXXVI, E. Wolf (Ed.), Elsevier, Amsterdam (1996).

[38] I. Bialinicki-Birula, Phys. Rev. Lett. 80, 5247 (1998).

[39] P. R. Holland, The Quantum Theory of Motion, Cambridge University Press, Cambridge (1993). 
[40] R. D. Prosser, Int. J. Theor. Phys. 15, 169 (1976).

[41] R. D. Prosser Int. J. Theor. Phys. 15, 181 (1976).

[42] M. Davidović, A. S. Sanz, D. Arsenović, M. Božić, and S. Miret-Artés, Phys. Scr. T135, 014009 (2009).

[43] C.-C. Chou and R. E. Wyatt, Phys. Scr. 830, 65403 (2011).

[44] J. D. Jackson, Classical Electrodynamics, Wiley, New York (1998) 3rd Ed.

[45] P. Ghose, A. S. Majumdar, S. Guha, and J. Sau, Phys. Lett. A 290, 205 (2001).

[46] M. Božić, M. Davidović, T. L. Dimitrova, S. Miret-Artés, A. S. Sanz, and A. Weis, J. Russ. Laser Res. 31, 117 (2010).

[47] M. Davidović, A. S. Sanz, M. Božić, D. Arsenović, and D. Dimić, Phys. Scr. T153, 014015 (2013).

[48] M. Davidović and A. S. Sanz, Europhysics News 44(6), 33 (2013).

[49] M. F. Pusey, J. Barrett, and T. Rudolph, Nature Phys. 8, 475 (2012).

[50] A. S. Sanz, F. Borondo, and S. Miret-Artés, J. Phys.: Condens. Matt. 14, 6109 (2002).

[51] M. Gondran and A. Gondran, Am. J. Phys. 78, 598 (2010).

[52] D. Bohm and B. J. Hiley, The Undivided Universe: An Ontological Interpretation of Quantum Theory, Routledge, London, New York (1993).

[53] P. G. Merli, G. F. Missiroli, and G. Pozzi, Am. J. Phys. 44, 306 (1976).

[54] H. Rauch and S. Werner, Neutron Interferometry: Lessons on Experimental Quantum Mechanics, Clarendon, Oxford (2000).

[55] P. R. Berman (Ed.) Atom Interferometry, Academic, New York (1997). 